\title{
Infrastructure planning and topology optimization for reliable mobile big data transmission under cloud radio access networks
}

\author{
Xiang Hou, Bin Lin, Rongxi He* and Xudong Wang
}

\begin{abstract}
With the development of user-centered and environment sensing technology of 5G, large capacity and ubiquitous coverage and massive data collection and processing will bring new challenges in wireless networks. The cloud radio access network (C-RAN) has been envisioned to provide a new wireless architecture for reliable transmission of mobile big data. In this paper, we focus on network planning deployment issue based on the optical mixed diet (OMD) technology. Specifically, the ring and spur topology optimization (RSTO) problem under the C-RAN architecture is investigated. The RSTO problem is formulated as a generic integer linear program (ILP) which can optimally (i) minimize the network deploying cost; (ii) identify the locations of Remote Radio Units (RRUs) and optical add-drop multiplexers (OADMs); (iii) identify the association relations between RRUs and OADMs; and (iv) satisfy the mobile coverage requirements so as to allow the mobile big data to be transmitted through the RRUs. We propose a new heuristic algorithm based on C-RAN architecture. Numerical results validate the ILP formulation and show the performance benefits of the proposed algorithm in terms of efficiency and effectiveness against Gurobi, which is an ILP solver. In numerical studies, we also demonstrate the performance benefits of the incorporation of CoMP technology in terms of total deployment cost reduction.
\end{abstract}

Keywords: Mobile big data, C-RAN, Integer linear program (ILP), Heuristic algorithm

\section{Introduction}

Nowadays, the information society has stepped into a new era, in which the mobile Internet, cloud computing, big data, and Internet of Things are the leading technologies [1]. The big data has main features: volume, variety, velocity, and value [2]. Thus, future wireless networks are required to provide the ability to store and process the large data.

Wireless access network is facing new challenges, such as network deployment, resource management, mobile data security, and network consumption. To deal with these challenges, a new type the radio access network architecture named cloud radio access network (C-RAN) has been proposed in [3].

\footnotetext{
* Correspondence: hrx@dlmu.edu.cn

College of Information Science and Technology, Dalian Maritime University, Dalian 116026, China
}

The C-RAN has four "C" characteristics, i.e., clean, centralized processing, collaborative radio, and realtime cloud radio access network. C-RAN is viewed as an architecture evolution based on distributed base stations (BSs). The C-RAN architecture breaks down the traditional BS into a base band unit (BBU) and Remote Radio Units (RRUs). The BBU is a digital unit that implements the MAC, PHY, and antenna array system functionality. The BBU is often located in a remote site such as a central office ( $\mathrm{CO}$ ) and thus the energy, real estate, and security costs are minimized. The RRU can be mounted either outdoor or indoor such as on poles, sides of buildings, or anywhere power and a broadband connection exist, making installation less costly and easier. The RRU is typically connected to the BBU using fibers. C-RAN adopts CoMP technology to reduce interference, lower power consumption, and improve spectral efficiency in [4] and [5]. 
Due to the requirement of a high-volume, variety, and non-uniform distribution of the big data in time domains and space domains, wireless access networks must have high reliability. For example, many applications of online banking, mobile payment, and remote business are generated on smart phones. Thus, the networks are required to transmit data much more reliably.

Different from the tree and branch topology, the fiber can transmit the signals between the BBU and the optical add-drop multiplexers (OADMs) bidirectionally in the ring and spur topology as shown in Fig. 1. When one of the fibers is interrupted, the other fibers could continue to achieve optical signal transmission. Thus, the ring and spur topology can avoid the network paralysis caused by fiber interruption [6].

In order to increase the utilization of the optical fiber, optical mixed diet (OMD) mode is proposed. In the OMD mode, the BBU pool supplies a pair of specific wavelengths to transmit the signal to the OADM. The OMD integrates the function of WDM. The BBU and a certain RRU are using a specific wavelength module and a plurality of RRU can share a pair of optical fibers; thus, the OMD mode can increase the utilization of the optical fiber.

In this paper, we focus on the ring and spur topology optimization (RSTO) problem under the C-RAN architecture. A generic integer linear programming (ILP) model is proposed to capture the nature of the RSTO problem. In this paper, we focus on network planning deployment issue based on the OMD technology.

The RSTO problem under the C-RAN architecture is investigated. The contributions of this paper are summarized as follows. (1) The RSTO problem is formulated as a generic ILP model which can optimally:(i) minimize the network deploying cost; (ii) identify the locations of RRUs and OADMs; (iii) identify the association relations between RRUs and OADMs; and (iv) satisfy the mobile coverage requirements so as to allow the mobile user access through the RRUs. (2) We also propose a new heuristic algorithm under C-RAN architecture. The algorithm can deploy the C-RAN architecture in small- and largescale scenarios. (3) Compared with the result of Gurobi in different scenarios, simulation results verify that the algorithm is correct and effective.

The remainder of the paper is organized as follows. In Section 2, we review the previous related work. In Section 3, the problem formulation is given, including the network model, given inputs, variables, constraints, and objective of the RSTO problem. The heuristic algorithm is presented in Section 4. Computational results and case studies are presented in Section 5. Finally, we conclude the paper in Section 6.

\section{Related work}

We review the related studies on network planning and placement. In [7], three types of architectures, namely optical fiber direct-drive, optical transmission network $(\mathrm{OTN})$ /wavelength division multiplexing (WDM), and unified passive optical network (Uni-PON) are proposed. These three models have been further elaborated in [8-10], respectively.

Our previous work has studied on the network planning and placement, which have direct influence on the future network deployment and long-term performance [11-15]. The problem formulated to determine the locations of communication network equipment is discussed as follows. Site planning of relay station GA-based solution in green wireless access network is proposed in [11]. An ILP

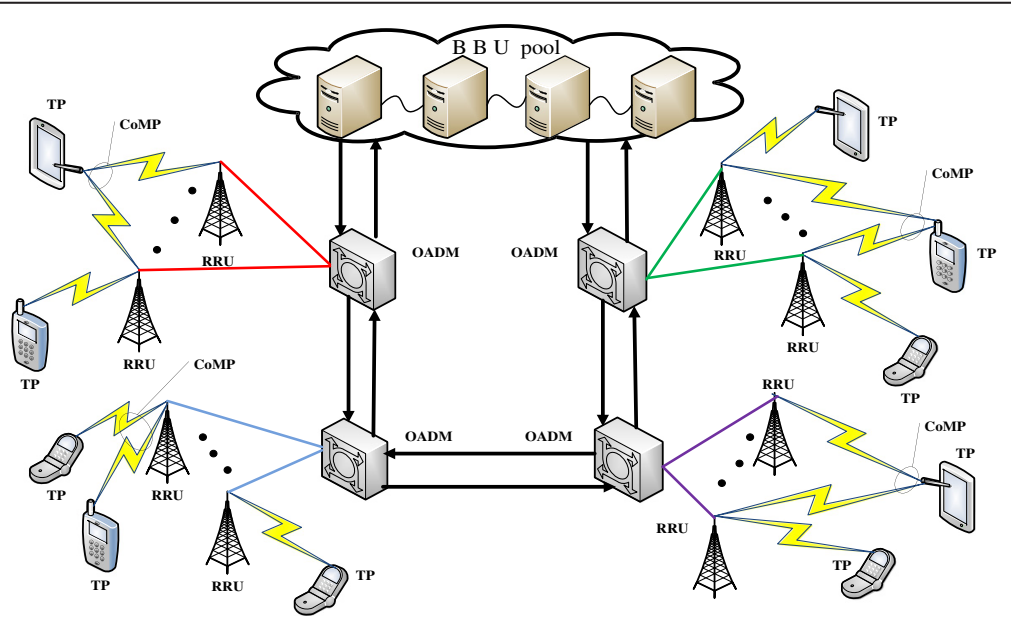

Fig. 1 The C-RAN network model based on the ring and spur topology 
model is formulated to maximize the throughput of an LTE network in [12]. Cascaded splitter placement and layout in long-reach PON are discussed in [13]. A dimensioning and site-planning optimization framework was proposed for integrated $\mathrm{PON}$ and wireless cooperative networks in [14]. An ILP model for the cascaded LRPON topology and network equipment placement, and network dimensioning are proposed in [15].

Other related works have studied on topology and placement optimization, such as [16] and [17]. A model for combined virtual core network function placement and topology optimization is proposed in [16]. In [17], the author presents some optimization problems in WMNs and different heuristic methods such as local search, genetic algorithms, and tabu search for solving them nearoptimally. Relayed-Based Heterogeneous Wireless Access Networks (RHWAN) is envisioned as the promising network architecture proposed in [18]. A hybrid wireless mesh network infrastructure which connects the smart meters of each consumer with the data aggregation points (DAP) is proposed in [19]. An optimal deployment model on Universal Data Aggregation Point (UDAP) is proposed in [20].

In this research, we choose the ring and spur topology optimization based on the OMD technology under the C-RAN architecture. The fiber can bidirectionally transmit the signal between the BBU and the OADMs; thus, it has high network survivability. Wireless access network is demanded to provide higher reliability in the big data era. To the best of our knowledge, there are few studies on the deployment and optimization for C-RAN infrastructure under the ring and spur topology, which is just the focus of our research.

\section{Network model and problem formulation}

\subsection{Network model}

The C-RAN network model consists of four network entities: BBU, OADM, RRU, and mobile stations (MSs), as shown Fig. 1. The OADMs and the RRUs are eligible to be deployed at certain outdoor potential sites (PSs) where uninterrupted power supply can be provided [5].
In order to construct the OADM ring, we define the concept of "flow" and related terms as shown in Fig. 2.

Term 1: Inflow: the directed path which represents the flow from BBU to OADM $\mathrm{O}_{k}$.

Term 2: Outflow: the directed path which represents the flow from $\mathrm{OADM}_{\mathrm{k}}$ to BBU.

Term 3: Inflow path: the path starts from BBU and ends at $\mathrm{OADM}_{k}$.

Term 4: Outflow path: the path starts from $\mathrm{OADM}_{k}$ and ends at the BBU.

Without loss of generality, we assume that the MSs are homogeneously distributed in the area of interest (AOI), which yields a uniform distribution on the traffic demand in the AOI. We define that a site is covered under the C-RAN architecture if the received radio signal strength at the site is above a given threshold level (receiver sensitivity). Small-scale fading is not explicitly included in the system model since a long-term planning and design is targeted. To test the mobile coverage, we define test points (TPs) within the AOI. The TPs are also used for testing the received power intensity for MSs from the associated RRU. In Fig. 1, the locations of TPs and PSs for both OADM and RRU are also illustrated.

\subsection{Problem statement}

The RSTO problem can be stated as follows. Let $G$ $=(\Omega: E)$, where $\Omega$ is the set of the nodes and $E$ is the set of the direct edge/links. The BBU is denoted as the node $B . \Omega$ is partitioned into four parts, i.e., the set of TP, the set of OADM, the set of RRU, and the set of BBU, which are denoted as $\Omega_{\mathrm{TB}}, \Omega_{\mathrm{OADM}}, \Omega_{\mathrm{RRU}}$, and $\Omega_{\mathrm{B}}$, i.e., $\Omega=\Omega_{\mathrm{TP}} \cup \Omega_{\mathrm{RRU}} \cup \Omega_{\mathrm{OADM}} \cup \Omega_{\mathrm{B}}$.

\subsubsection{Given}

(1) The set of fixed TPs, which are the customer premises

(2) The set of PSs for deploying RRUs and OADMs

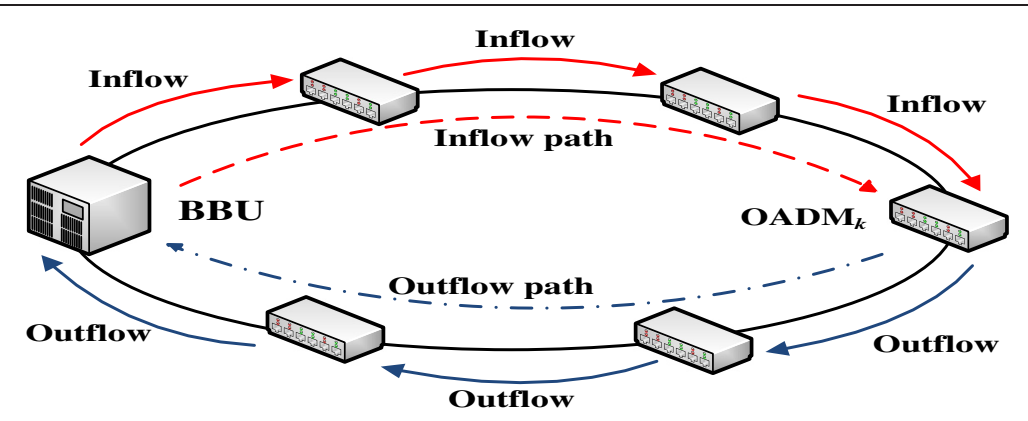

Fig. 2 Flow on the OADM ring 
(3) The location of the BBU

(4) The cost per unit length of fiber $(\$ / \mathrm{km}) C^{f}$, including fiber purchase and deployment cost.

(5) The Manhattan distance between node $i$ and node $j\left(d_{i j}\right)$.

(6) The cost of RRU $\left(C^{\mathrm{R}}\right)$ and OADM $\left(C^{\mathrm{O}}\right)$.

(7) The maximal transmit power of a RRU

(8) The minimal signal-to-noise-ratio (SNR) requirement for an MS

\subsection{Variable}

(1) The PS ${ }_{m}$ is selected to place a RRU if $a_{m}=1$; otherwise, $a_{m}=0$.

(2) The $\mathrm{PS}_{t}$ is selected to place an OADM if $b_{t}=1$; otherwise, $b_{t}=0$.

(3) The MS at $\mathrm{TP}_{n}$ is associated with the RRU if $z_{m n}=1$; otherwise, $z_{m n}=0$.

(4) There is a directed link from node $i \in \Omega_{\mathrm{B}} \cup \Omega_{\mathrm{OADM}}$ to node $j \in \Omega_{\mathrm{B}} \cup \Omega_{\mathrm{RRU}} \cup \Omega_{\mathrm{OADM}}$ if $e_{i j}=1$; otherwise, $e_{i j}=0$.

(5) The $\mathrm{TP}_{n}$ is covered if $q_{n}=1$; otherwise, $q_{n}=0$.

(6) $e_{i j}$ is on the directed flow from the BBU to $\mathrm{OADM}_{k}$ if $g_{i j}^{k}=1$; otherwise, $g_{i j}^{k}=0$.

(7) $e_{i j}$ is on the directed flow from the $\mathrm{OADM}_{k}$ to $\mathrm{BBU}$ if $h_{i j}^{k}=1$; otherwise $h_{i j}^{k}=0$.

\subsection{Constraints}

(1) The mobile coverage requirement should be satisfied, i.e., the coverage ratio should be larger than a predefined value.

(2)The SNR for an MS located at each TP should be larger than a SNR threshold.

(3)Each TP is covered by at least one RRU.

(4) The OADM ring consists the BBU and the selected OADMs.

(5) There must be at least a RRU to connect to the selected OADM.

(6) The RRU can cover the MS with CoMP technology.

(7) The double-flow control is used to establish the mathematical model.

(8)If the PS is selected to place an OADM on the OADM ring, there must be an input path and output path.

\subsection{Objective}

The objective is to minimize the infrastructure deployment cost of the whole C-RAN network. Table 1 lists important symbols for the problem formulation.

\subsection{Problem formulation}

(RSTO) objective:

Table 1 Definitions of Symbols in the RSTO problem

\begin{tabular}{|c|c|}
\hline Symbol & Definition \\
\hline$\Omega_{\mathrm{B}}$ & The set of the BBU \\
\hline$\Omega_{\mathrm{OADM}}$ & The set of the OADMs \\
\hline$\Omega_{\mathrm{RRU}}$ & The set of the RRUs \\
\hline$\Omega_{\mathrm{TP}}$ & The set of the TPs \\
\hline$T$ & The number of OADMs in the network \\
\hline M & The number of RRUs in the network \\
\hline N & The number of TPs in the network \\
\hline$d_{i j}$ & The distance between node $i$ and node $j$ \\
\hline$P$ & The maximal transmit power of a RRU \\
\hline$\xi$ & The average normalized thermal noise in $\mathrm{AOI}$ \\
\hline a & The path loss exponent \\
\hline$s_{0}$ & The minimal SNR requirement for each TP \\
\hline A & The location incidence vector, $A=\left(a_{m}\right)_{1 \times M}$ \\
\hline B & The location incidence vector, $B=\left(b_{t}\right)_{1 \times T}$ \\
\hline Q & A TP coverage incidence vector, $Q=\left(q_{n}\right)_{1 \times N}$ \\
\hline Z & RRU-TP association matrix $Z=\left(z_{m n}\right)_{M \times N}$ \\
\hline E & BBU-OADM-RRU association matrix, $E=\left(e_{i j}\right)_{(T+1)} \times(M+T)$ \\
\hline$D_{\text {MAX }}$ & The maximal distance of OMD-RRU that can be deployed in the network \\
\hline$H^{K}$ & $\mathrm{OADM}_{k}-\mathrm{BBU}$ flow association matrix $H^{K}=\left(h_{i j}^{k}\right)_{(1+T) \times(T+M)}$ \\
\hline$G^{K}$ & $\mathrm{BBU}-\mathrm{OADM}_{k}$ flow association matrix $G^{K}=\left(g_{i j}^{k}\right)_{(1+T) \times(T+M)}$ \\
\hline
\end{tabular}




$$
\text { Minimize } \quad C=C^{\mathrm{F}}+C^{\mathrm{RRU}}+C^{\mathrm{OADM}}
$$

where

$$
\begin{aligned}
& C^{\mathrm{F}}=\sum_{i \in \Omega_{\mathrm{B}}}\left(e_{i j}+e_{i j}\right) \cdot d_{i j}+C^{f} \sum_{i \in \Omega_{\mathrm{OADM}}} e_{i j} \cdot d_{i j} \\
& \quad+C^{f} \sum_{i \in \Omega_{\mathrm{OADM}}} \sum_{j \in \Omega_{\mathrm{RRU}}} e_{i j} \cdot d_{i j} \\
& C^{\mathrm{RRU}}=C^{R} \sum_{m \in \Omega_{\mathrm{RRU}}} a_{m} \\
& C^{\mathrm{OADM}}=C^{O} \sum_{t \in \Omega_{\mathrm{OADM}}} b_{t}
\end{aligned}
$$

Equation (1.1) is to minimize the total deployment cost of the C-RAN network, including the cost of fibers, the cost of RRUs, and the cost of OADMs.

Subject to:

$$
\begin{aligned}
& \sum_{m \in \Omega_{\mathrm{RRU}}} z_{m n} \geq 1, \forall n \in \Omega_{\mathrm{TP}} \\
& z_{m n} \leq a_{n}, \forall m \in \Omega_{\mathrm{RRU}}, \forall n \in \Omega_{\mathrm{TP}} \\
& e_{i j} \leq b_{i}, \forall i \in \Omega_{\mathrm{OADM}}, \forall j \in \Omega_{\mathrm{RRU}}
\end{aligned}
$$

Constraint (1.2) stipulates that each TP is covered by at least one RRU, and it ensures the CoMP feature of C-RAN. Constraint (1.3) ensures that the PS is selected to place a RRU if $a_{m}=1$. Constraint (1.4) ensures that the PS is selected to place an OADM if $b_{i}=1$.

$$
\begin{aligned}
& \sum_{j \in \Omega_{\mathrm{OADM}}} e_{i j}=1, \forall i \in \Omega_{\mathrm{B}} \\
& \sum_{i \in \Omega_{\mathrm{OADM}}} e_{i j}=1, \forall j \in \Omega_{\mathrm{B}}
\end{aligned}
$$

Constraints (1.5) and (1.6) ensure that the BBU can be connected to the OADM ring. Constraint (1.5) stipulates that the BBU has just only one OADM as BBU's output. Constraint (1.6) stipulates that the BBU has just only one OADM as BBU's input.

$$
\begin{aligned}
& \sum_{i \in \Omega_{B} \cup \Omega_{\mathrm{OADM}}} e_{i j}=a_{j}, \forall j \in \Omega_{\mathrm{OADM}}, i \neq j \\
& \sum_{j \in \Omega_{B} \cup \Omega_{\mathrm{OADM}}} e_{i j}=a_{j}, \forall i \in \Omega_{\mathrm{OADM}}, i \neq j
\end{aligned}
$$

Constraints (1.7) and (1.8) ensure that an OADM can be connected to the OADM ring. Constraint (1.7) ensures that if the PS is selected to place an OADM on the OADM ring, then anther OADM serves as an output terminal in the OADM ring. Constraint (1.8) ensures that if the PS is selected to place an OADM on the OADM ring, then anther OADM serves as an input terminal in the OADM ring. $\sum_{i \in \Omega_{\mathrm{OADM}}} e_{i j}=a_{j}, \forall j \in \Omega_{\mathrm{RRU}}$

$$
\sum_{j \in \Omega_{\mathrm{RRU}}} e_{i j} \geq b_{i}, \forall i \in \Omega_{\mathrm{OADM}}
$$

Constraint (1.9) ensures that if the PS is selected to place an OADM on the OADM ring, then at least one RRU should be connected to the selected OADM, if $a_{j}=$ 1. Constraint (1.10) ensures that RRUs can be connected to the OADM, if $b_{i}=1$.

$$
e_{i j} \cdot d_{i j} \leq D_{\max }, \forall i \in \Omega_{\mathrm{OADM}}, \forall j \in \Omega_{\mathrm{RRU}}
$$

Constraint (1.11) sets an upper bound on the fiber length between an OADM and a RRU in C-RAN network.

$$
g_{i j}^{k} \leq e_{i j}, \forall i \in \Omega_{\mathrm{B}} \cup \Omega_{\mathrm{OADM}}, \forall j \in \Omega_{\mathrm{OADM}}, k \in \Omega_{\mathrm{OADM}}, i \neq k
$$

$$
h_{i j}^{k} \leq e_{i j}, \forall i \in \Omega_{\mathrm{OADM}}, \forall j \in \Omega_{B} \cup \Omega_{\mathrm{OADM}}, k \in \Omega_{\mathrm{OADM}}, j \neq k
$$

Constraint (1.12) stipulates that only when $e_{i j}=1, e_{i j}$ probably exists in the inflow path from $\mathrm{BBU}$ to $\mathrm{OADM}_{k}$. Constraint (1.13) stipulates that only when $e_{i j}$ $=1, e_{i j}$ probably exists in the outflow path from the $\mathrm{BBU}$ to $\mathrm{OADM}_{k}$.

$$
g_{i j}^{k} \leq b_{k}, \forall i \in \Omega_{\mathrm{B}} \cup \Omega_{\mathrm{OADM}}, \forall j \in \Omega_{\mathrm{OADM}}, k \in \Omega_{\mathrm{OADM}}, i \neq k
$$

$$
h_{i j}^{k} \leq b_{k}, \forall i \in \Omega_{\mathrm{OADM}}, \forall j \in \Omega_{B} \cup \Omega_{\mathrm{OADM}}, k \in \Omega_{\mathrm{OADM}}, j \neq k
$$

Constraints (1.14) and (1.15) ensure only when $\mathrm{OADM}_{i}$ and $\mathrm{OADM}_{j}$ exist firstly, then there probably exists the path between the BBU and $\mathrm{OADM}_{k}$.

$$
\begin{aligned}
& \sum_{j \in \Omega_{\mathrm{OADM}}} g_{i j}^{k}=b_{k}, \forall k \in \Omega_{\mathrm{OADM}}, i \in \Omega_{\mathrm{B}} \\
& \sum_{i \in \Omega_{\mathrm{OADM}}} h_{i j}^{k}=b_{k}, \quad \forall k \in \Omega_{\mathrm{OADM}}, j \in \Omega_{\mathrm{B}}
\end{aligned}
$$

For all OADMs, constraints (1.16) and (1.17) ensure where the inflow path must start from the $\mathrm{BBU}$, and the outflow path must end at the BBU.

$$
\begin{aligned}
& \sum_{i \in \Omega_{B} \cup \Omega_{\mathrm{OADM}}} g_{i k}^{k}=b_{k}, \forall k \in \Omega_{\mathrm{OADM}}, i \neq k \\
& \sum_{j \in \Omega_{B} \cup \Omega_{\mathrm{OADM}}} h_{k j}^{k}=b_{k}, \forall k \in \Omega_{\mathrm{OADM}}, j \neq k
\end{aligned}
$$

Constraints (1.18) and (1.19) stipulate that for $\mathrm{OADM}_{k}$, its inflow path must end at $\mathrm{OADM}_{k}$, and its outflow must start from $\mathrm{OADM}_{k}$. 


$$
\begin{aligned}
& \sum_{j \in \Omega_{\mathrm{OADM}}} \sum_{k \in \Omega_{\mathrm{OADM}}} g_{i j}^{k}=\sum_{k \in \Omega_{\mathrm{OADM}}} b_{k}, i \in \Omega_{\mathrm{B}} \\
& \sum_{i \in \Omega_{\mathrm{OADM}}} \sum_{k \in \Omega_{\mathrm{OADM}}} h_{i j}^{k}=\sum_{k \in \Omega_{\mathrm{OADM}}} b_{k}, j \in \Omega_{\mathrm{B}}
\end{aligned}
$$

Constraints (1.20) and (1.21) ensure that only when $\mathrm{OADM}_{k}$ is selected to place on the OADM ring, then $\mathrm{OADM}_{k}$ has an inflow path and an outflow path.

$$
\begin{gathered}
\sum_{i \in \Omega_{B} \cup \Omega_{\mathrm{OADM}}} g_{i j}^{k}=\sum_{\substack{i \neq j \in \Omega_{\mathrm{OADM}} \\
i \neq m, i \neq j \neq k}} g_{j m}^{k}, \forall j, k \in \Omega_{\mathrm{OADM}}, \\
\sum_{i \in \Omega_{\mathrm{OADM}}} h_{i j}^{k}=\sum_{\substack{i \neq j \in \Omega_{\mathrm{B}} \cup \Omega_{\mathrm{OADM}} \\
i \neq m, i \neq j \neq k}} h_{j m}^{k}, \forall j, k \in \Omega_{\mathrm{OADM}},
\end{gathered}
$$

Constraint (1.22) stipulates that if $\mathrm{OADM}_{j}$ is on the path from $\mathrm{BBU}$ to $\mathrm{OADM}_{k}$, then $\mathrm{OADM}_{j}$ has an input path and an output path. Also, there is an inflow path that belongs to the path from $B B U$ to $\mathrm{OADM}_{j}$. Constraint (1.23) stipulates that if $\mathrm{OADM}_{j}$ is on the path from BBU to $\mathrm{OADM}_{k}$, then $\mathrm{OADM}_{j}$ has an input path and an output path. Also, there is an outflow path that belongs to the path from BBU to $\mathrm{OADM}_{k}$.

$$
\begin{aligned}
& g_{i j}^{k}+h_{i j}^{k} \leq 1, \forall i, j, k \in \Omega_{\mathrm{OADM}}, i \neq j \neq k \\
& e_{i j}+e_{j i} \leq 1, \forall i \in \Omega_{B} \cup \Omega_{\mathrm{OADM}}, \forall j \in \Omega_{\mathrm{OADM}}, i \neq j \\
& \frac{1}{\xi} \sum_{m \in \Omega_{\mathrm{RRU}}} \frac{z_{m n} \times P}{d_{m n}^{\alpha}} \geq s_{0}, \forall n \in \Omega_{\mathrm{TP}} \\
& q_{n} \geq \frac{1}{\sum_{m \in \Omega_{\mathrm{RRU}}} \frac{P}{\xi d_{m n}^{\alpha}}-s_{0}}\left(\sum_{m \in \Omega_{\mathrm{RRU}}} \frac{P}{\xi d_{m n}^{\alpha}}-s_{0}\right) \\
& 1-q_{n} \geq \frac{1}{\sum_{m \in \Omega_{\mathrm{RRU}}} \frac{P}{\xi d_{m n}^{\alpha}}-s_{0}}\left(s_{0}-\sum_{m \in \Omega_{\mathrm{RRU}}} \frac{P}{\xi d_{m n}^{\alpha}}\right) \\
& \frac{1}{N} \sum_{n \in \Omega_{\mathrm{TP}}} q_{n} \geq \eta \times 100 \%
\end{aligned}
$$

For $\mathrm{OADM}_{k}$, constraint (1.24) stipulates that the same path cannot exist in the inflow path and outflow path at the same time. Constraint (1.25) stipulates that the path is directional, which means only one direction can be chosen. Constraint (1.26) ensures that the TP satisfies the constraint of the minimum SNR requirement. Constraints (1.27)-(1.29) stipulate the definition of $\mathrm{Q}$.

$$
\begin{aligned}
& a_{m} \in\{0,1\}, \forall m \in \Omega_{\mathrm{RRU}}, b_{t} \in\{0,1\}, \forall t \in \Omega_{\mathrm{OADM}}, \\
& q_{n} \in\{0,1\}, \forall n \in \Omega_{\mathrm{TP}} \\
& z_{m n} \in\{0,1\}, \forall m \in \Omega_{\mathrm{RRU}}, \forall n \in \Omega_{\mathrm{TP}} \\
& e_{i j} \in\{0,1\}, \forall i \in \Omega_{\mathrm{B}} \cup \Omega_{\mathrm{OADM}}, \forall j \in \Omega_{\mathrm{OADM}} \cup \Omega_{\mathrm{RRU}} \\
& g_{i j}^{k} \in\{0,1\}, \forall i \in \Omega_{\mathrm{B}} \cup \Omega_{\mathrm{OADM}}, \forall j \in \Omega_{\mathrm{OADM}}, k \in \Omega_{\mathrm{OADM}}, i \neq k \\
& h_{i j}^{k} \in\{0,1\}, \forall i \in \Omega_{\mathrm{OADM}}, \forall j \in \Omega_{\mathrm{B}} \cup \Omega_{\mathrm{OADM}}, k \in \Omega_{\mathrm{OADM}}, j \neq k
\end{aligned}
$$

Constraints (1.30)-(1.34) state the each entry $A, B, Q$, $Z, E, H^{K}$, and $G^{K}$ is binary.

\section{Heuristic algorithm}

\subsection{Algorithm description}

The problem formulation is given in the previous section, including the network model, given inputs, variables, constraints, and objective of the RSTO problem. However, with the continuous expansion of network size, solving the above RSTO formulation is not an easy task especially for large-scale networks due to the exponential growing nature of ILP. To make the design more scalable for largesize networks, in this section, we propose a new heuristic algorithm.

The design and process of this new heuristic algorithm is mainly concluding some steps as follows:

(1) Initialization of system parameters.

(2)RRU selection. This step achieves the coverage TP through candidate RRU.

(3) OADM selection.

(4) Construct the C-RAN architecture based on the ring and spur topology.

(5)C-RAN layout (including the location of selected RRUs and OADMs, the optical link between RRUs and OADMs, the optical ring of OADMs and BBU, the association between RRUs and OADMs), total deployment cost.

Now, we introduce the pseudocode of this the heuristic algorithm, and then we give an application based on this heuristic algorithm.

The pseudocode of the heuristic algorithm is outlined below. 


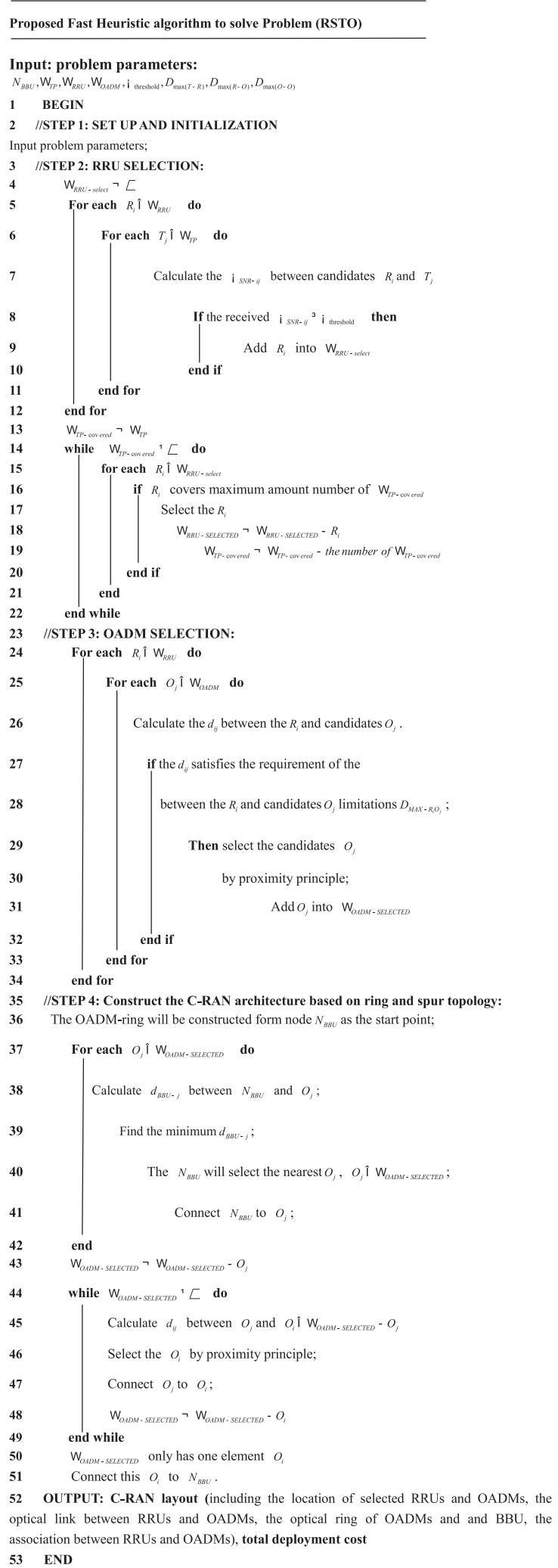

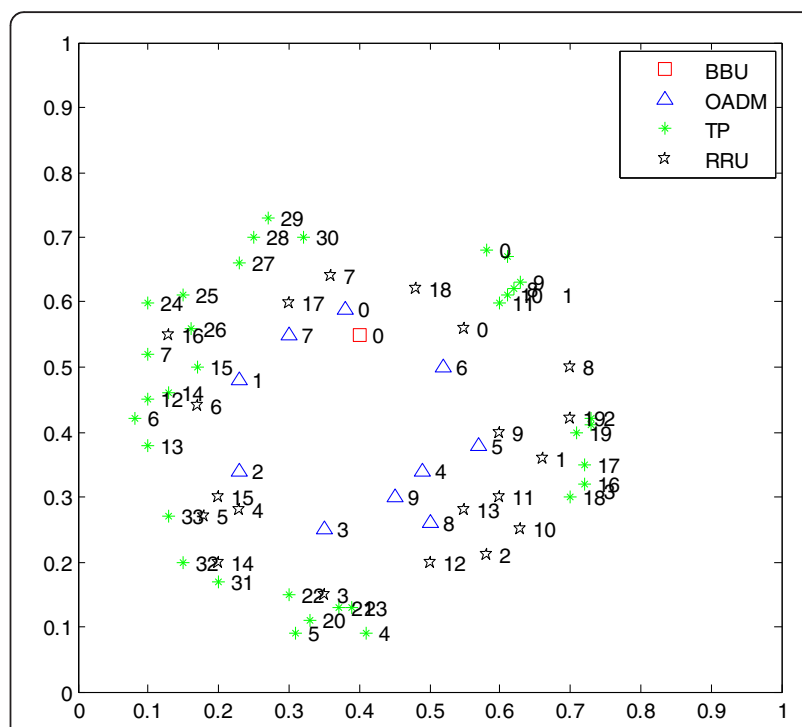

Fig. 3 The layout and setting of scenario 0 before topology optimization

The proposed heuristic is stated in detail as follows:

Step 1(Lines 1-2): Initialization. Firstly, a plurality of TPs, potential sites for deploying RRUs, and

OADMs are set in an AOI after an actual survey. A two-dimensional coordinate graph is established on the basis of the latitude and longitude of the actual positions of TPs, and RRU candidates and OADM candidates are import into this two-dimensional coordinate graph based on their actual location of the latitude and longitude. The distances between TPs and RRU candidates (i.e., parameter $d_{\mathrm{T}-\mathrm{R}}$ ), RRU candidates and OADM candidates (i.e., parameter

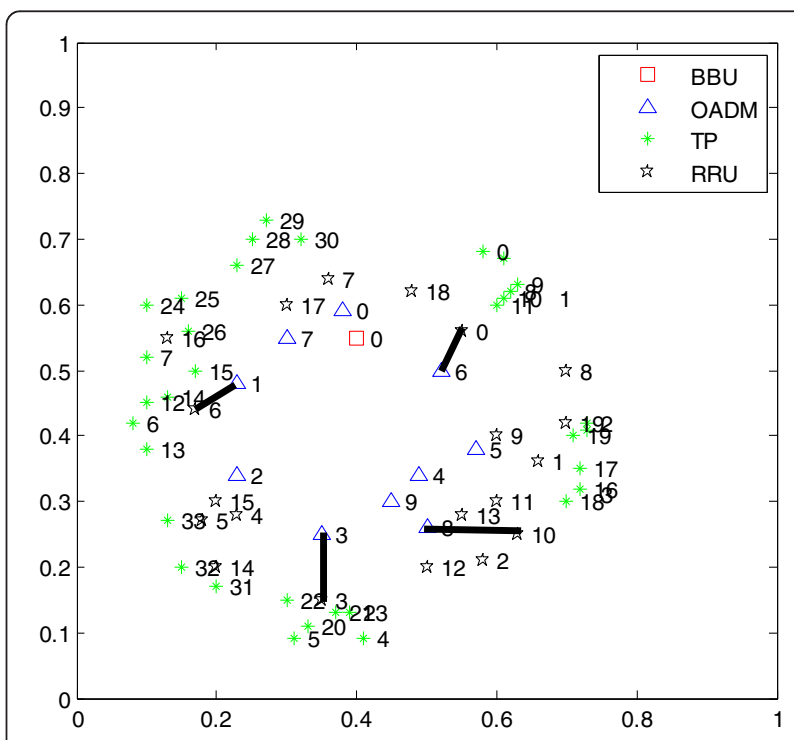

Fig. 4 RRU selection process in scenario 0 
$\left.d_{\mathrm{R}-\mathrm{O}}\right)$, OADM candidates and OADM candidates (i.e., parameter $d_{\mathrm{O}-\mathrm{O}}$ ) are calculated and stored, respectively. The parameters of $N_{\mathrm{BBU}}, \Omega_{\mathrm{TP}}, \Omega_{\mathrm{RRU}}$,

$\Omega_{\mathrm{OADM}}, \quad$ threshold $, D_{\max (\mathrm{T}-\mathrm{R})}, D_{\max (\mathrm{R}-\mathrm{O})}, D_{\max (\mathrm{O}-\mathrm{O})}$ are set according to the requirement of actual deployment scenarios.

Step 2 (Lines 3-22): RRU selection. The goal of this step is to make each TP covered by one candidate RRU. First, we calculate the SNR between the TPs and candidates RRUs. If the received SNR at a TP form a candidate RRU that satisfies the minimum SNR threshold, then we select the RRU which covers the maximum amount of the TPs, then add

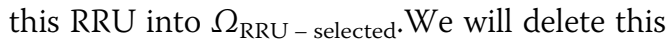
already selected RRU and these TPs which are covered by selected RRU. The TPs will select the RRU in turn according to the calculated TP numbers covered by the candidate RRU in descending order The candidates can be selected until all TPs are covered by the candidate RRU.

Step 3 (Lines 23-34): OADM selection. The goal of this step is to set up the optical links between the candidate OADM and all the selected RRUs at step 2. We calculate the distances between the selected RRUs and candidate OADMs. If the distance satisfies the requirement of the selected RRU and candidate OADM limitations (i.e., parameter $\left.D_{\max (\mathrm{R}-\mathrm{O})}\right)$, we select the candidate OADM by a proximity principle. Then, we will add this OADM into $\Omega_{\mathrm{OADM}}$ - selected. If each selected RRU could select an OADM which satisfies the requirement of the distance limitations, then these OADMs will be added into $\Omega_{\mathrm{OADM}}$ - selected. The loop will end until

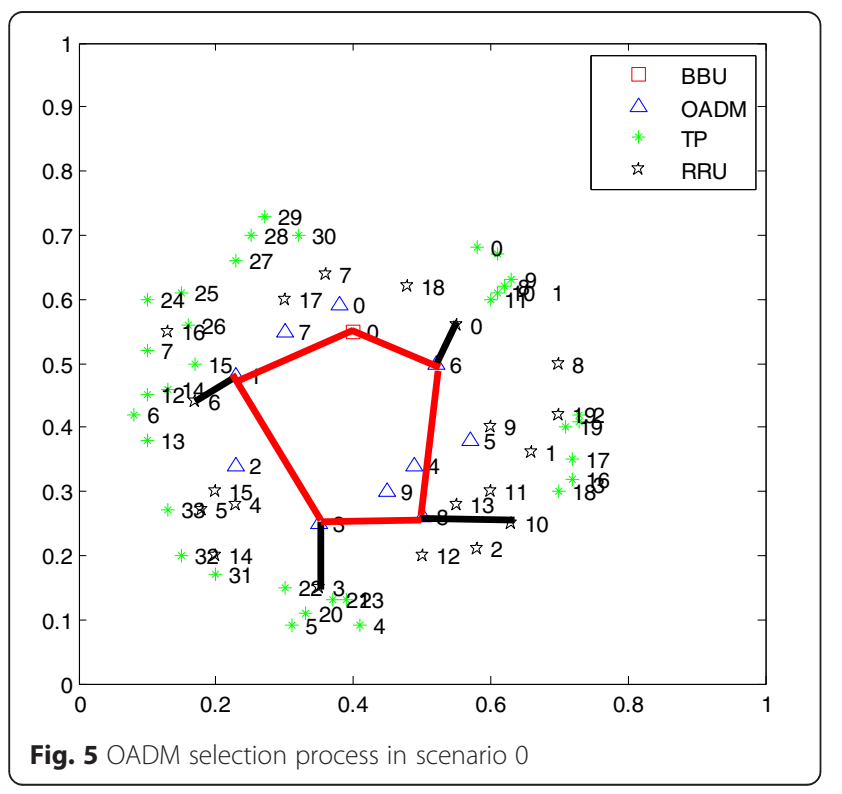

Table 2 C-RAN component cost

\begin{tabular}{ll}
\hline Component & Cost (gcu) \\
\hline Fiber $(/ \mathrm{km})$ & 1 \\
Burying fiber $(/ \mathrm{km})$ & 50 \\
RRU & 16 \\
OADM & 50 \\
\hline
\end{tabular}

all the selected RRU at step 2 could connect to an OADM. The optical links between the selected RRU at step 2 and OADM can be set up.

Step 4 (Lines 35-51): Construct the C-RAN architecture based on the ring and spur topology. The goal of this step is to construct the OADM ring. The ring will be constructed from the BBU as the start point. Firstly, we calculate the distance (i.e., parameter $\left.d_{\mathrm{BBU}-\mathrm{O}}\right)$ between the BBU and the selected OADMs (i.e., parameter $\Omega_{\mathrm{OADM}}$ - selected $)$. Secondly, we find the minimum $d_{\mathrm{BBU}-O_{j}}$ for all $O_{j}$ and connect the $\mathrm{BBU}$ to the corresponding $O_{j}$, then remove this

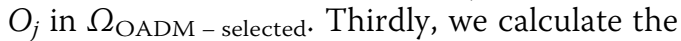
distance $\left(d_{O_{j}-O_{i}}\right)$ between $O_{j}$ and $O_{i}\left(O_{i} \in \Omega_{\mathrm{OADM}-}\right.$ selected $\left.-O_{j}\right)$, then we also find the minimum distance and connect $O_{j}$ to $O_{i}$. The loop will end until the $\Omega_{\mathrm{OADM}}$ - selected has only one element $O_{i}$. Finally, we connect this $O_{i}$ to BBU, and the OADM ring can be constructed.

Finally, in lines 52-53, the heuristic outputs the layout solution (including the location of the selected RRUs and OADMs, the optical link between RRUs and OADMs, the optical ring of OADMs and BBU, the association between RRUs and OADMs) and total deployment cost.

\subsection{An example for the proposed fast heuristic algorithm} In this section, we use a small-scale scenario, denoted as scenario 0 , as an example to illustrate the procedure of the proposed algorithm. The parameter setting of scenario 0 is as follows: the set of TPs denotes $P_{T P}$, the candidate RRUs denote $P_{\text {RRU, and the candidate }}$ OADMs denote $P_{\text {OADM }}$ (see Fig. 3 ).

We defined a generic cost unit (gcu) [21] to simplify the evaluation of deployment costs in all the case studies.

Table 3 Simulation parameter settings

\begin{tabular}{ll}
\hline Parameter & Value \\
\hline$a$ & 2 \\
$P$ & $8 \mathrm{~W}$ \\
$S_{0}$ & $29 \mathrm{~dB}$ \\
$\xi$ & $2 \mathrm{~mW}$ \\
$D_{\text {MAX }}$ & $10 \mathrm{~km}$ \\
Scale & $20 \mathrm{~km}$ \\
\hline
\end{tabular}



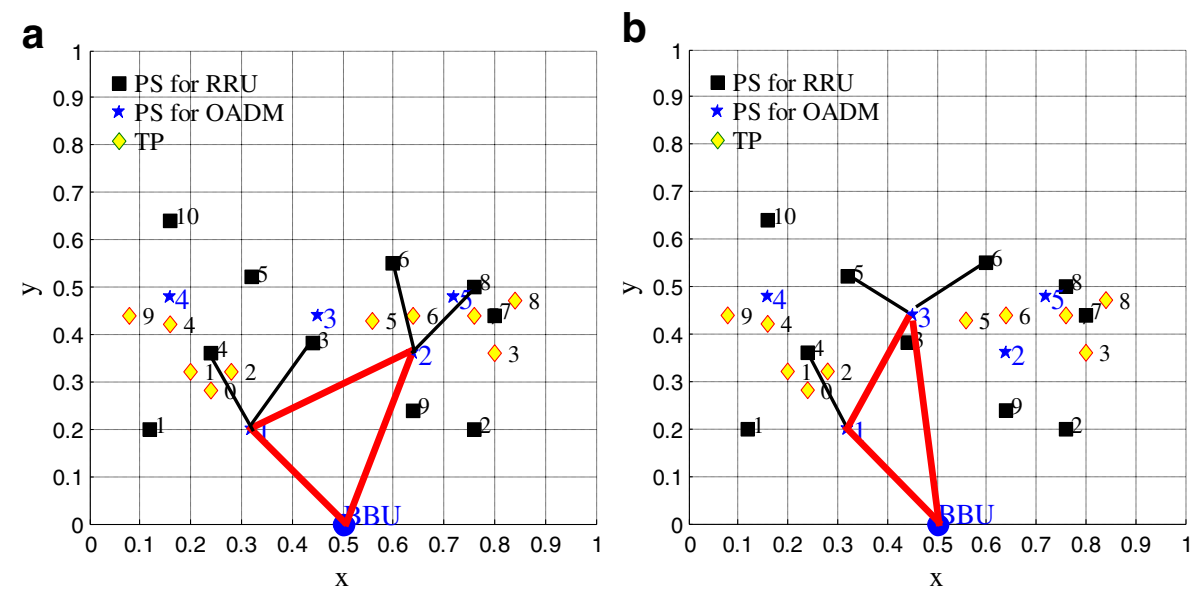

Fig. 6 Network layout results in scenario 1. a Without CoMP. b With CoMP

Step 1: Initialization.

Step 2: Achieve all of the TPs that can be covered by the candidate RRUs. In Fig. 3, the set of the candidate RRUs denotes $P_{\text {RRU }}=\left\{P_{\text {RRU }} 0, P_{\text {RRU }} 1, P_{\text {RRU }} 2, \cdots, P_{\text {RRU }} 19\right\}$. The set of the candidate OADMs denotes $P_{\text {OADM }}$ $=\left\{P_{\text {OADM }} 0, P_{\text {OADM }} 1, \cdots, P_{\text {OAdM }} 9\right\}$. The set of TPs denotes $P_{\mathrm{TP}}=\left\{P_{\mathrm{TP}} 0, P_{\mathrm{TP}} 1, P_{\mathrm{TP}} 2, \cdots, P_{\mathrm{TP}} 34\right\}$. All of the TPs can be covered by the four RRUs, $P_{\mathrm{RRU}} 10, P_{\mathrm{RRU}} 0, P_{\mathrm{RrU}} 3, P_{\mathrm{RRU}} 6$. In specific, 17 TPs can be covered by $P_{\text {RRU }} 10 ; 6$ TPs can be covered by $P_{\text {RRU }} 0 ; 6$ TPs can be covered by $P_{\text {RRU }} 3$; and 6 TPs can be covered by $P_{\text {RRU }} 6$.

Step 3: For all the selected RRUs, the optical link between the candidate OADMs and the selected RRUs will be connected at this step. Instead of using a proximity principle in selecting the candidate OADMs, we also conclude the distance between the RRUs and OADMs. In Fig. 4, there are four OADMs which can be connected to the RRUs, which are denoted as $P_{\text {OADM }} 1, P_{\text {OADM }} 3$ , Pódm $6, P_{\mathrm{OAdm}} 8$. In specific, the $P_{\mathrm{rRu}} 6$ can be connected to $P_{\text {OAdm }} 1$, the $P_{\text {RRu }} 3$ can be connected to $P_{\text {OAdm }} 3$, the $P_{\text {RRU }} 0$ can be connected to $P_{\mathrm{OADM}} 6$, and the $P_{\mathrm{RRU}} 10$ can be connected to $P_{\mathrm{OADM}} 8$.

Step 4: Achieve the C-RAN architecture based on ring and spur. In this step, the ring topology will start form $\mathrm{BBU}$, then the BBU will select the nearest OADM. We

Table 4 Comparison of RSTO results with and without COMP in scenario 1

\begin{tabular}{lll}
\hline Scenario 1 & $\begin{array}{l}\text { Without CoMP } \\
\left(s_{0}=23 \mathrm{~dB}\right)\end{array}$ & $\begin{array}{l}\text { With CoMP } \\
\left(s_{0}=23 \mathrm{~dB}\right)\end{array}$ \\
\hline $\begin{array}{l}\text { Selected number of PSs for RRU } \\
\text { placement }\end{array}$ & 4 RRUs & 3 RRUs \\
$\begin{array}{l}\text { Obj. value (gcu) } \\
\text { Compute time (s) }\end{array}$ & 1760.8 & 1543.8 \\
Optimality Gap & 0.02 & 0.03 \\
\hline
\end{tabular}

marked this OADM as OADM-s(1). If the OADM-s(1) connects to the RRUs, then the OAMD satisfies the OADM ring. In Fig. 5, the BBU connects to $P_{\mathrm{OADM}} 6$, then

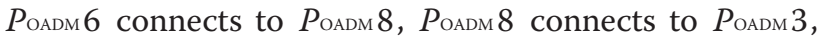
$P_{\text {OADM }} 3$ connects to $P_{\text {OAdM }} 1$, and $P_{\text {OAdM }} 1$ connects to $\mathrm{BBU}$. So, the ring and spur topology can be constructed at this step.

Finally, output the optical link between RRUs and OADMs, the optical link between OADM and BBU, the association between RRUs and OADMs (see Fig. 5).

Step 5: Compute the total deployment cost, computing time in this ring and spur topology.

In scenario 0, the total deployment cost is $1824.43 \mathrm{gcu}$. The topology running time is $1.308 \mathrm{~s}$. The layout and total deployment cost results of the ring and spur topology under C-RAN is the same as the result obtained with Gurobi which will be introduced later in next section.

\section{Numerical analysis}

\subsection{Parameter settings}

We implement the optimization model of the previous section and solve the RSTO problem using Gurobi Optimizer [22], which is a state-of-the-art ILP solver.

Table 5 Problem size of simulated scenarios

\begin{tabular}{|c|c|c|c|c|}
\hline \multirow[t]{2}{*}{ Scenario } & \multicolumn{4}{|c|}{ Number of nodes } \\
\hline & $\mathrm{TP}$ & \multicolumn{2}{|c|}{ RRU candidates } & OADM candidates \\
\hline । & 100 & \multicolumn{2}{|l|}{40} & 20 \\
\hline$\|$ & 500 & \multicolumn{2}{|l|}{120} & 50 \\
\hline \multirow[t]{2}{*}{ III } & 900 & \multicolumn{2}{|l|}{190} & 80 \\
\hline & Constraints & Variables & Nonzero & Ave. memory \\
\hline I & 3058 & 2419 & 8423 & $127.59 \mathrm{MB}$ \\
\hline$\|$ & 9371 & 6843 & 26418 & $736.74 \mathrm{MB}$ \\
\hline III & 24873 & 18397 & 72629 & $7851.38 \mathrm{MB}$ \\
\hline
\end{tabular}


Table 6 Comparison analysis of small-scale scenarios

\begin{tabular}{|c|c|c|c|c|c|c|}
\hline \multirow{2}{*}{$\begin{array}{l}\text { Scenario } \\
\text { Scheme }\end{array}$} & \multicolumn{2}{|l|}{1} & \multicolumn{2}{|l|}{$\|$} & \multicolumn{2}{|l|}{ III } \\
\hline & Gurobi & Heuristic & $\overline{\text { Gurobi }}$ & Heuristic & $\overline{\text { Gurobi }}$ & Heuristic \\
\hline $\begin{array}{l}\text { Obj. value } \\
\text { (gcu) }\end{array}$ & 3458.12 & 3614.47 & 4672.35 & 4928.84 & 6028.96 & 6375.14 \\
\hline $\begin{array}{l}\text { Computing } \\
\text { time (s) }\end{array}$ & 17.24 & 2.07 & 46.39 & 4.15 & 130.12 & 6.34 \\
\hline $\begin{array}{l}\text { Optimality } \\
\text { gap }\end{array}$ & 4.529 & & 5.48 & & 5.74 & \\
\hline
\end{tabular}

Gurobi is designed to exploit modern multi-core processors and the performance is proved to be superior to CPLEX [23]. Without loss of generality, the Manhattan distance of two nodes is used to represent the corresponding edge length of the fiber deployment route, and all the fiber segment links have the same weighted deployment costs.

Tables 2 and 3 show the component cost of C-RAN and experimental parameters, respectively. We still use the generic cost unit (gcu) [21] to simplify the evaluation of deployment costs in the case studies.

In scenario 0 , we have validated the solvability of (RSTO) formulation and the effectiveness of our proposed heuristic. To further investigate the effect of CoMP in the CRAN planning strategy, we compare the resulting network layout configuration with and without CoMP technology in scenario 1 as shown in Fig. 6. We can observe the difference between Figs. 6a, b. The number of the selected RRUs in Fig. 6a is more than that in Fig. 6b. Table 4 shows the corresponding computing result of network optimal layout in scenario 1. It compares the objective value with and without CoMP. Obviously, the total cost of the network will be increased without CoMP. In other words, the incorporation of CoMP technology can lead to a significant cost reduction for C-RAN.

\subsection{Numerical analysis of small-scale scenarios}

Considering that the scale and configuration of a network placement may affect the performance of a network planning, we investigate a number of small-scale scenarios. Table 5 lists only three of the simulated scenarios with an increasing network scale. In the simulations, we randomly select PSs and TPs in the area. The problem size, the average computation time, the optimization gap, and the objective values in three

Table 7 Problem size of simulated large scenarios

\begin{tabular}{llll}
\hline Scenario & \multicolumn{2}{l}{ Number of nodes } \\
\cline { 2 - 4 } & TP & RRU candidates & OADM candidates \\
\hline IV & 1500 & 300 & 150 \\
V & 2500 & 450 & 230 \\
\hline
\end{tabular}

Table 8 Comparison analysis for large scenarios

\begin{tabular}{llrllr}
\hline Scenario & IV & & & \\
\cline { 2 - 3 } \cline { 5 - 6 } Scheme & Gurobi & Heuristic & & Gurobi & Heuristic \\
\hline Obj. value $(\mathrm{gcu})$ & No result & 8127.67 & & No result & 10342.14 \\
Computing time (s) & & 7.15 & & 8.63 \\
\hline
\end{tabular}

scenarios are shown in Tables 5 and 6. The results demonstrate that our proposed heuristic algorithm can obtain the solution network layout much more effectively than Gurobi, and the optimality gap of the objective value between our heuristic and Gurobi is less than $6 \%$. In other words, the proposed heuristic can obtain the near-optimal solution highly efficiently.

\subsection{Numerical analysis of larger-scale scenarios}

To investigate the performance of proposed heuristic in larger-scale scenarios, we further simulated scenarios with thousands of nodes as listed in Table 7. The problem size, the average computation time, and the objective values in the scenarios are shown in Table 8.

From Table 8, Gurobi can hardly find the optimal result with the increase of the network scale; however, the heuristic algorithm can still obtain the solution effectively and efficiently.

Therefore, we can conclude that the RSTO-formulationbased Gurobi solution scheme are more suitable for smaller-scale C-RAN network layout planning, and our proposed heuristic algorithm could be suitable for realworld large-scale C-RAN network layout planning in practice.

\subsection{Comparison of the tree and branch and ring and spur topology}

Comparing Table 9 with Table 10, we can observe that:

(i) The deployment cost of the ring topology is relatively higher than that of the tree topology due to the deployment of bidirectional optical fiber.

(ii) In respect to the computing time, the ring topology is shorter than the tree topology. In the tree topology, it needs to make restrictions to every path from the BBU to the RRUs, but in the ring topology, it only needs to make restrictions to the path in the OADM ring.

Table 9 The network scale of scenarios IX, X, and XI

\begin{tabular}{llll}
\hline Scenario & $I X$ & $X$ & $X \mid$ \\
\hline PS for OA/OADMs & 5 & 5 & 5 \\
PS for RRUs & 20 & 30 & 50 \\
The number of TPs & 20 & 40 & 100 \\
\hline
\end{tabular}


Table 10 Comparison of the tree and ring topology

\begin{tabular}{|c|c|c|c|c|c|c|}
\hline \multirow{2}{*}{$\begin{array}{l}\text { Scenario } \\
\text { Scheme }\end{array}$} & \multicolumn{2}{|l|}{ IX } & \multicolumn{2}{|l|}{$x$} & \multicolumn{2}{|l|}{$X I$} \\
\hline & Tree & Ring & Tree & Ring & Tree & Ring \\
\hline $\begin{array}{l}\text { Total cost } \\
\text { (unit, gcu) }\end{array}$ & 1834.46 & 2208.12 & 2436.54 & 2895.03 & 3269.07 & 3890.01 \\
\hline $\begin{array}{l}\text { Computing } \\
\text { time }\end{array}$ & 2.09 & 0.34 & 5.41 & 0.87 & 7.65 & 1.21 \\
\hline
\end{tabular}

\section{Conclusions}

C-RAN provides a promising wireless architecture for reliable transmission of mobile big data. In this paper, we study the fundamental problem of topology optimization based on the more reliable string and spur topology. We formulate the RSTO problem to minimize the total deployment cost of the C-RAN network. We have examined the proposed RSTO problem via extensive case studies in term of its feasibility and scalability. Furthermore, we propose a new heuristic algorithm to obtain the near-optimal C-RAN network layout effectively and efficiently, especially suitable for a large-scale C-RAN network scenario in practice. In numerical studies, we also demonstrate the performance benefits of incorporation of CoMP technology in terms of total deployment cost reduction.

\section{Competing interests}

The authors declare that they have no competing interests.

\section{Acknowledgements}

This study is sponsored by the National Science Foundation of China (NSFC) No. 61371091, No. 61171175, and No. 61301228; the National Science Foundation of Liaoning Province No. 2014025001; the Program for Liaoning Excellent Talents in University (LNET) No. LJQ2013054; and the Fundamental Research Funds for Central Universities under grant No. 3132015045.

Received: 31 December 2015 Accepted: 24 April 2016

Published online: 03 May 2016

\section{References}

1. P Zhang, Q Cui, Y Hou, J Xu, Opportunities and challenges of wireless networks in the era of mobile big data. Journal of China Science 60, 433-438 (2015)

2. The 34th Statistical Report of the Department of Internet in China. Chinese Internet Network Information Centre, 2014.

3. Q Lei, Z Zhang, F Cheng, $5 \mathrm{G}$ radio access network architecture based on CRAN. Telecommunication Science 1, 1-10 (2015)

4. X. Wang. C-RAN: the road towards green RAN. China Communication Journal, 2010

5. K. Chen. C-RAN White Paper, version 2.5. China Mobile Research Institute,2011

6. MA Esmail, H Fathallah, Fiber Fault Management and Protection Solution for Ring-and-Spur WDM/TDM Long-Reach PON. 2011 IEEE Global Telecommunications Conference, 2011, pp. 1-5

7. http://www.huawei.com. Accessed 1 Oct 2011

8. A design algorithm for ring topology centralized-radio-access-network Paper presented at the Optical Networks Design and Modelling (ONDM) Conference, Brest, France, 2013

9. K. Ramantas, K. Vlachos, G. Ellinas, A. Hadjiantonis. A converged optical wireless architecture for mobile backhaul networks. Paper presented at the Optical Networks Design and Modelling (ONDM) Conference, Brest, France, pp. 155-160, 2013

10. L Kazovsky, SW Wong, T Ayhan, KM Albeyoglu, MRN Ribeiro, A Shastri, Hybrid optical-wireless access networks. Proc. IEEE 100(5), 1197-1225 (2012)
11. B Lin, L Lin, Site planning of relay station in green wireless access networks: a genetic algorithm approach, in Soft Computing and Pattern Recognition (SoCPaR), Dalian, China, 2011, pp. 167-172

12. $\mathrm{B}$ Lin, $\mathrm{PH} H \mathrm{Ho}, \mathrm{L}$ Xie, $\mathrm{X}$ Shen, J Tapolcai, Optimal relay station placement in broadband wireless access networks. IEEE Transaction on Mobile Computing 9(2), 259-269 (2010)

13. B Lin, Y Tian, Energy and Radiation-Aware Base Station Placement in EcoSustainable LTE Networks. 2nd International Conference on Electronics, in Communication and Control (ICECC2012), 2012, pp. 3162-3165

14. B Lin, L Lin, PH Ho, Cascaded Splitter Topology Optimization in LRPONs. International Conference on Communications (ICC), Ottawa, Canada, 2012 pp. 3105-3109

15. L Lin, B Lin, PH Ho, Power-Aware Optimization Modeling for Cost-Effective LRPON Infrastructure Deployment. 21st International Conference on Software, Telecommunications and Computer Networks (SoftCOM), 2013, pp. 1-5

16. A Baumgartner, VS Reddy, T Bauschert, Mobile Core Network Virtualization: A Model for Combined Virtual Core Network Function Placement and Topology Optimization. 2015 1st IEEE conference on Network Softwarization (NetSoft), London, 2015, pp. 1-9

17. A Barolli, F Xhafa, M Takizawa, Optimization Problems and Resolution Methods for Node Placement in Wireless Mesh Network. 2011 14th International Conference on Network-Based Information Systems (NBiS), Tirana, 2011, pp. 126-134

18. B Lin, J Guo, R He, T Yang, Infrastructure Deployment and Dimensioning of Relay-Based Heterogeneous Wireless Access Networks for Green Intelligent Transportation. The 2nd conference on Catalysis (ICC 2014), Suzhou, 2014, pp. 447-460

19. E Inga-Ortega, A Peralta-Sevilla, RC Hincapie, Infrastructure Deployment and Dimensioning of Relay-Based Heterogeneous Wireless Access Networks for Green Intelligent Transportation. 2015 IEEE PES on Innovative Smart Grid Technologies Latin America (ISGT LATAM), Montevideo, 2015, pp. 30-35

20. A Peralta-Sevilla, E Inga, R Cumbal, R Hincapie, Optimal Dimensioning of FiWi Networks Over Advanced Metering Infrastructure for the Smart Grid. 2015 IEEE Colombian Conference on Communications and Computing (COLCOM), Popayan, 2015, pp. 1-6

21. DAR Chaves, EA Barboza, CJA Bastos-Filho, A Multi-Objective Approach to Design All-Optical and Translucent Optical Networks Considering CapEx and QoT. 14th International Conference on Transparent Optical Networks (ICTON) 49(1), 1-4 (2012)

22. Gurobi Optimizer 4.6, Gurobi Optimization Inc., 2012.

23. Gurobi Optimizer, State of the Art Mathematical ProgrammingN Solver, [online], http://www.gurobi.com/.

\section{Submit your manuscript to a SpringerOpen ${ }^{\mathcal{O}}$ journal and benefit from:}

- Convenient online submission

- Rigorous peer review

- Immediate publication on acceptance

- Open access: articles freely available online

- High visibility within the field

- Retaining the copyright to your article

Submit your next manuscript at $\boldsymbol{s p r i n g e r o p e n . c o m ~}$ 